\title{
International Collaboration for Improved Public Health Emergency Preparedness and Response in India
}

\author{
Obaghe Edeghere ${ }^{* 1}$, Giri Shankar ${ }^{1}$, Alastair Bartholomew ${ }^{1}$, SriKrishna Ramachandra ${ }^{2}$, \\ Vivek Singh ${ }^{2}$, Pradeep Banandur ${ }^{3}$, Linda Parr ${ }^{1}$, Kenny Yap ${ }^{1}$, Babatunde Olowokure ${ }^{1}$ and \\ Sue Ibbotson ${ }^{1}$
}

${ }^{1}$ The Health Protection Agency, Birmingham, United Kingdom; ${ }^{2}$ Indian Institute of Public Health, Hyderabad, India; ${ }^{3}$ Rajarajeswari Medical College and Hospital, Bangalore, India

\section{Objective}

This project aimed to contribute to ongoing efforts to improve the capability and capacity to undertake disease surveillance and Emergency Preparedness and Response (EPR) activities in India. The main outcome measure was to empower a cadre of trainers through the inter-related streams of training \& education to enhance knowledge and skills and the development of collaborative networks in the regions.

\section{Introduction}

The International Health Regulations (IHR) 2005, provides a framework that supports efforts to improve global health security and requires that, member states develop and strengthen systems and capacity for disease surveillance and detection and response to public health threats. To contribute to this global agenda, an international collaborative comprising of personnel from the Health Protection Agency, West Midlands, United Kingdom (HPA); the Indian Institute of Public Health (IIPH), Hyderabad, Andhra Pradesh (AP) state, India and the Department of Community Medicine, Rajarajeswari Medical College and Hospital (RRMCH), Bangalore, Karnataka state, India was established with funding from the HPA Global Health Fund to deliver the objectives stated above.

\section{Methods}

In 2010, the project partners jointly developed training materials on applied Epidemiology \& Disease Surveillance and EPR using existing HPA material as the foundation. Over a 2 year period, a total of two training courses per year were planned for each of the two locations in India. Courses were designed to be delivered through didactic lectures, simulation exercises, workshops and group discussions at the two locations, namely Bangalore and Hyderabad. The target audience included senior state level programme officers, District Medical and Health Officers, postgraduate students, academic and research staff from Community Medicine departments and staff from the collaborating institutions.

Course modules were formally evaluated by participants using structured questionnaires and an external evaluator. Debrief sessions were also arranged after each course to review the key lessons and identify areas for improvement.

In addition, staff exchanges of up to six weeks duration were planned during which public health specialists from both countries would spend time observing health protection systems/processes in their host country.

\section{Results}

During January 2010 to December 2011, a total of seven $(n=7)$ training courses were delivered in Bangalore and Hyderabad with ap- proximately 231 public health personnel in attendance over the period. Participants comprised of 128 personnel representing 74 organisations in 41 districts ( 22 districts from AP) at the Hyderabad location and 103 personnel from 14 organisations (30 districts) at the Bangalore location.

Course participants evaluated the content of the courses favourably with the majority $(92 \%)$ rating the course modules as excellent or good. External evaluation of the courses was also favourable with several aspects of the course rated as good or excellent. IIPH and RRMC continue to deliver the courses and in the state of Karnataka, some participants at the EPR course were chosen by the health ministry to be part of Rapid Response Teams at District levels.

Two public health specialists from each of the Indian organisations spent six (6) weeks in the United Kingdom as part of the planned staff exchanges. The exchanges were assessed to have been successful with important areas for future collaboration identified including proposals to jointly develop an Emergency Preparedness and Response Manual for the Indian Public Health audience.

\section{Conclusions}

The implementation and maintenance of effective and sustainable systems to ensure global health security relies on a well-trained public health workforce in member states. This innovative collaborative project has gone some way towards meeting its objective of establishing and supporting a cadre of trainers to ensure sustainable improvement in public health capacity and capability in India. After the initial (training) phase of the project that was completely funded by the HPA, the partner organisations in India have worked to sustain and further develop the core objectives of this project. As a further step, course materials developed as part of this project will be used to provide a framework upon which e-learning material and postgraduate modules will be developed in each of these institutions in India.

\section{Keywords}

Surveillance; Training; EPR; IHR

\section{Acknowledgments}

Leanne Baker - HPA, UK

Krishna Gayathri - IIPH, Hyderabad

*Obaghe Edeghere

E-mail: obaghe.edeghere@hpa.org.uk 